\title{
Lipid II: A central component in bacterial cell wall synthesis and a target for antibiotics
}

\author{
Ben de Kruijff*, Vincent van Dam, Eefjan Breukink \\ Chemical Biology and Organic Chemistry, Utrecht University, Padualaan 8, Utrecht, The Netherlands
}

\begin{abstract}
A B S T R A C T
The bacterial cell wall is mainly composed of peptidoglycan, which is a three-dimensional network of long aminosugar strands located on the exterior of the cytoplasmic membrane. These strands consist of alternating MurNAc and GlcNAc units and are interlinked to each other via peptide moieties that are attached to the MurNAc residues. Peptidoglycan subunits are assembled on the cytoplasmic side of the bacterial membrane on a polyisoprenoid anchor and one of the key components in the synthesis of peptidoglycan is Lipid II. Being essential for bacterial cell survival, it forms an attractive target for antibacterial compounds such as vancomycin and several lantibiotics. Lipid II consists of one GlcNAcMurNAc-pentapeptide subunit linked to a polyiosoprenoid anchor 11 subunits long via a pyrophosphate linker. This review focuses on this special molecule and addresses three questions. First, why are special lipid carriers as polyprenols used in the assembly of peptidoglycan? Secondly, how is Lipid II translocated across the bacterial cytoplasmic membrane? And finally, how is Lipid II used as a receptor for lantibiotics to kill bacteria?
\end{abstract}

(c) 2008 Elsevier Ltd. All rights reserved.

\section{Introduction}

The bacterial cell wall is a unique structure. It consists mainly of a peptidoglycan polymer network located at the outside of the cytosolic membrane and it provides strength and shape to bacteria. A schematic presentation of the peptidoglycan layer and the biosynthesis of its building blocks is presented in Fig. 1. The units consist of the disaccharide GlcNAc and MurNAc to which a pentapeptide is attached. This unit is assembled on the cytosolic side of the membrane on a bactoprenol hydrocarbon chain via a pyrophosphate linker. The enzymes MraY and MurG catalyze the final assembly steps, resulting in the formation of Lipid II. This molecule translocates to the periplasmic side of the membrane, where the penicillin-binding proteins (PBPs) catalyze the insertion of peptidoglycan uniting into a growing cell wall. The lipid anchor carrying the pyrophosphate is shuttled back to the cytosolic side of the membrane, where it can be reused (after dephosphorylation to the mono-phosphate form) for the next round of synthesis.

All enzymes involved in peptidoglycan synthesis are known and various mechanistic aspects of the pathway are understood. This is not surprising since this pathway forms a prime target for antibiotics, of which penicillin and vancomycin probably are the best-known examples (Fig. 1). Both these antibiotics act on the periplasmic steps of the pathway involving Lipid II. This is also true for a subset of lantibiotics, which also target Lipid II and

\footnotetext{
* Corresponding author. Tel.: +31302531607; fax: +31302533969.

E-mail address: b.dekruijff@uu.nl (B. de Kruijff).
}

which will be discussed later. Despite considerable knowledge of cell wall synthesis several key questions remained unanswered so far. It is the aim of this paper to identify some of these key questions and to discuss and speculate on possible answers.

The specific questions to be addressed are:

1. Why did nature choose polyprenols as lipid anchors for the peptidoglycan units?

2. How are Lipid II molecules translocated from the cytosolic to the periplasmic leaflet of the membrane?

3. How can lantibiotics in such a versatile manner use Lipid II as receptor to kill bacteria?

Before these questions are addressed, it is useful to provide some more in-depth insight into Lipid II.

\section{Lipid II occurrence and properties}

Lipid II is a minor component of the bacterial cytoplasmic membrane. Estimates for Gram-positive bacteria typically give values below $1 \mathrm{~mol} \%$ of the membrane phospholipids [1]. This small amount of Lipid II is responsible for the fast growing bacterial cell wall. It can be estimated that the entire Lipid II cycle will take somewhat less than $1 \mathrm{~s}$, which immediately implies that most steps of the cycle will have even higher turnover numbers.

The chemical structure of Lipid II and a space-filling model are presented in Fig. 2. It shows a large hydrophilic head group linked via a pyrophosphate to a very long bactoprenol chain. Given the 


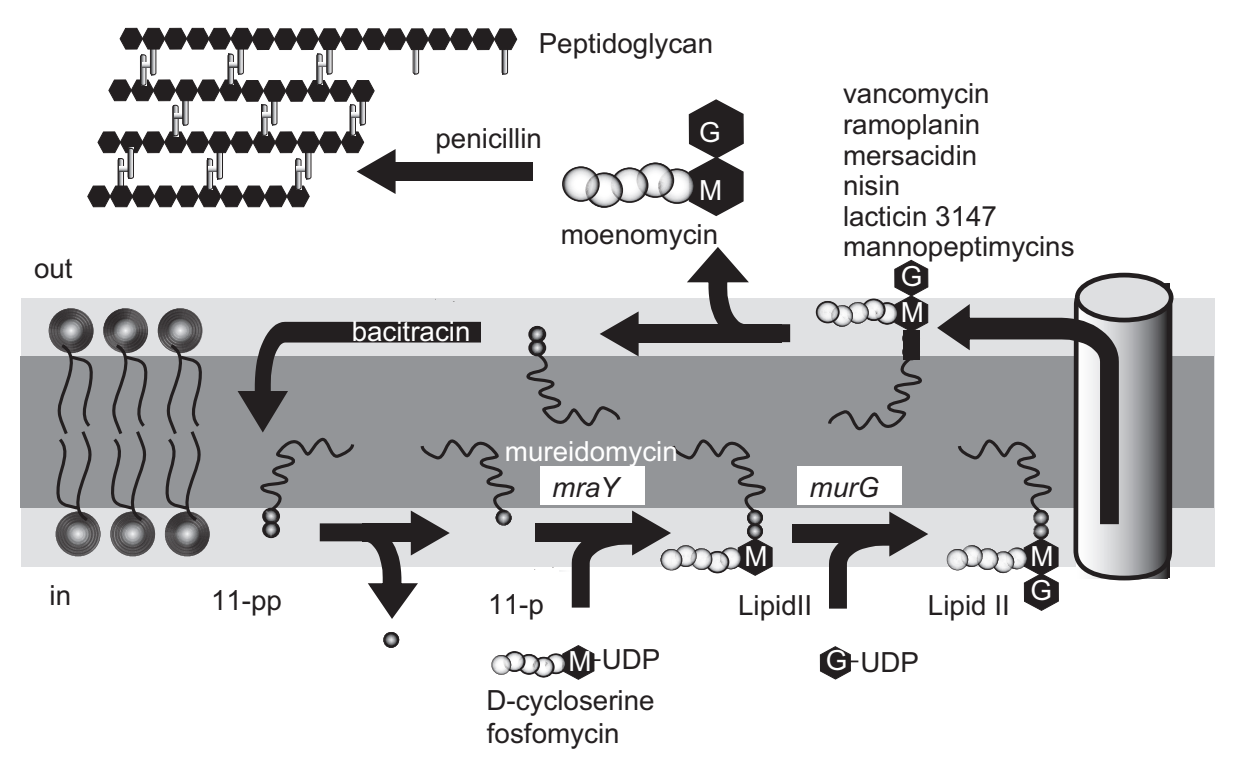

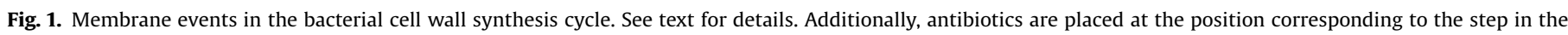

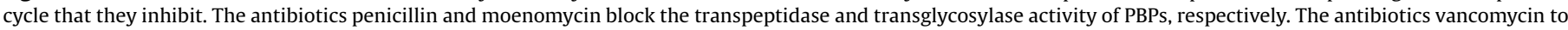

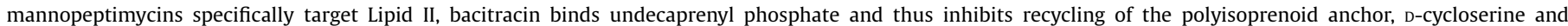
fosfomycin inhibit the synthesis of UDP-MurNAc-pentapeptide and mureidomycin specifically inhibits MraY.
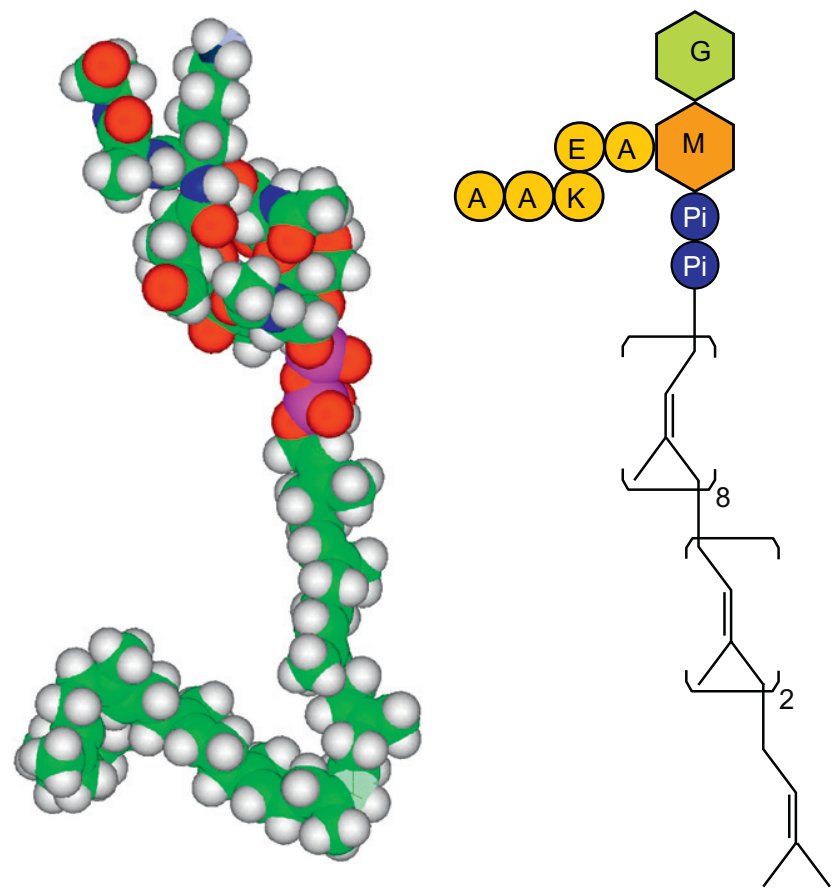

Fig. 2. Chemical structure and space-filling model of Lipid II. The specific configurations of the isoprene units are shown in the right structure, as well as the typical linkage (via the side chain) between the glutamate and lysine of the pentapeptide.

complexity of the molecule it is not surprising that the total chemical synthesis was only recently accomplished. Fortunately, a semi-synthetic production method of Lipid II has been described [2] that allows flexible production of Lipid II and its analogues in quantities sufficient for biochemical and biophysical research.

Incorporation of Lipid II in supported bilayers of phosphatidylcholine revealed that the bactoprenol chain must be fluid, because Lipid II partitions in fluid domains, present in mixed solid/fluid bilayers. This was observed by atomic force microscopy (AFM) using the lantibiotic nisin, which binds specifically to Lipid II to visualize its location [3]. Consistent with this finding was the observation made by confocal fluorescence microscopy that NBD-labeled Lipid II was homogeneously distributed in GUVs of DOPC [4].

The AFM technique also revealed that the head group of Lipid II is soft and can be penetrated by the AFM probe [3]. This property of the molecule allowed insight into the dimensions of the head group, which was estimated to be $1.9 \mathrm{~nm}$ high as compared to the level of the phospholipid head group [3]. Given that the limiting area of the head group is $1.5 \mathrm{~nm}^{2}$ as observed in monolayer studies, the pentapeptide must have an upright position and be fully accessible to the aqueous phase [3].

Another intriguing property of Lipid II and several of its precursors is that it displays a pyrophosphate in the membrane interface. This could readily serve as a binding motive for the proteins involved in the Lipid II cycle. In the forthcoming sections it will be described that the pyrophosphate of Lipid II forms the binding motive for a class of lantibiotics.

\section{Why polyprenols as lipid anchors for the peptidoglycan units?}

Typical bacterial membrane lipids have alkyl chains as hydrophobic moieties. However, the bactoprenols, being polyisoprenoids, are rare but functionally very important constituents of the hydrophobic interior of the bacterial membrane. This immediately raises the question as to why bactoprenol acts as lipid anchor for the peptidoglycan unit instead of the more conventional alkyl chains. Before discussing this question it is useful to consider the biological processes that make use of polyprenol-linked precursors. Within the bacterial membrane the bactoprenols, next to their role in peptidoglycan synthesis, also fulfill carrier functions in the synthesis pathways of other complex (poly)saccharides such as the $\mathrm{O}$-antigen of LPS, oligosaccharides involved with N-linked protein glycosylation in Campylobacter jejuni or the teichoic acids of Grampositive bacteria [5-7].

Interestingly, a striking parallel can be noticed between bacterial peptidoglycan synthesis and $\mathrm{N}$-glycosylation in eukaryotic cells. $N$-glycosylation takes place on the luminal site of the endoplasmic reticulum and involves the transfer of a large oligosaccharide unit to specific asparigine residues during protein translocation [8-11]. The mannose-rich oligosaccharide is linked via a pyrophosphate to a long polyprenol chain of the dolichol 
type, which strikingly resembles the bactoprenol chain. Thus in both peptidoglycan synthesis and $\mathrm{N}$-glycosylation the precursors are minor-polyprenol-anchored (pyro)phosphate-coupled membrane components.

These examples suggest that building a carbohydrate containing biopolymer from a membrane is best done via a polyprenollinked precursor. But why is this the case? One possibility is that polyprenols are ancient lipids and nature decided to stick to these lipids for some specific processes like $N$-glycosylation or bacterial cell wall and outer membrane synthesis. In this scenario the alkyl chains became the evolution-preferred main components of the hydrophobic membrane interior. Perhaps more obvious possibilities can be provided on analyzing in some more detail the processes the biopolymer precursors are involved in. The biopolymer unit in all cases has to be built into the growing polymer in a dynamic and flexible manner.

The long polyprenol chain could act as a spring-like structure, allowing flexible positioning at some variable distance from the membrane of the cargo to the enzymatic machines, incorporating it into the growing polymer. That the polyprenol chain will have special elastic properties is immediately apparent from the material properties of rubber, which is a polyisoprenoid. But also considerations on the molecular dimensions of the polyprenol lipid anchors suggest that they have spring-like properties. The polyprenol chains are, in extended form, longer than the thickness of the entire membrane bilayer. It is therefore highly likely that the polyprenol chain is curled up to some extent like a spring. Preliminary MD simulations support this view and suggest that the polyprenol chain on average has an L-shape with a vertical part, a quasi-helical chain and flexible horizontal part that is located between the two monolayers (unpublished results).

Another possibility is that the polyprenol chain (possibly in conjunction with the polyphosphate group) could act as a recognition motive for proteins that are involved in the formation of the biopolymer. This could localize the fluid lipid at its functional location.

Further possibilities can be derived from considerations of the biosynthesis of the membrane precursor. In all cases the polyprenollinked precursors are formed in a cyclic process (Fig. 1 shows this for the Lipid II synthesis pathway). These are multi-step enzymatic processes taking place on both sides of the membrane and thus involve at least two membrane translocation steps. The possibility that the bactoprenol chain could be involved in this process is considered in the next section.

\section{How are Lipid II molecules translocated from the cytosolic to the periplasmic side of the bacterial membrane?}

As a background to this question, first the current knowledge of transmembrane transport of the main bacterial membrane lipids will be given.

Since the biosynthesis of the main membrane lipids takes place at the cytosolic side of the membrane and the cells rapidly grow and divide, transmembrane transport must be a fast process. Indeed, all experimental studies that addressed this issue reported halftimes for transport of phospholipids in the order of minutes or less [12-15]. Biosynthesis and transport were found to be mechanistically not coupled and transport in $\rightarrow$ out was comparably rapid to transport out $\rightarrow$ in $[13,16]$. Transport of bacterial phospholipids across proteinfree bilayers of bacterial lipids is very slow [17]. It was thus concluded that phospholipid transport across the bacterial membrane must be protein mediated. Phospholipid transport is ATP or pmf independent [13]. So far no specific proteins responsible for this transport have been described, but instead a more general mechanism has been proposed. In this mechanism the hydrophobic transmembrane helical domains of membrane proteins themselves catalyze transport with single-spanning proteins, being more efficient than multispanning proteins $[18,19]$. This model was proposed for all biogenic membranes, including the endoplasmic reticulum, which show very similar characteristics for transmembrane transport of lipids as the bacterial system. Transmembrane-helices-catalyzed lipid flip-flop was found to be blocked by cholesterol [19], which provided an explanation for the lack of fast transmembrane transport of lipids in eukaryotic plasma membranes [20].

The high turnover numbers within the Lipid II cycle implicate that Lipid II transport across the bacterial membrane must also be rapid. Studies using model membranes demonstrated that translocation across a lipid bilayer is extremely slow and, in contrast to membrane phospholipids, is not stimulated by the presence of transmembrane helices [21], suggesting a more specific proteinmediated mechanism that is involved in phospholipid translocation.

MurG was found to catalyze the synthesis of Lipid II from its precursor Lipid I in model membranes, but the resulting Lipid II remained on the synthesis side of the bilayer, excluding the possibility that MurG itself is solely responsible for rapid translocation [21]. Consistent with this finding was the observation that Lipid II synthesis in the bacterial system is not obligatorily coupled to translocation, but must be mediated by a specialized protein machinery [21]. Transport was found to be ATP or pmf independent and appeared to be coupled to transglycosylation activities on the periplasmic side of the inner membrane [21].

Also for this step in the peptidoglycan synthesis pathway there is a striking parallel with $\mathrm{N}$-glycosylation of newly synthesized proteins in the endoplasmic reticulum. The dolichol-linked oligosaccharide is assembled on the cytosolic side of the ER after it is translocated via a specific proteinaceous system to the luminal side of the ER membrane, where it serves as a substrate for $N$-glycosylation [22].

The results described above exclude the possibility that the bactoprenol chain itself, with its special properties, is sufficient for rapid transmembrane transport of Lipid II. Other studies came to the same conclusion for the dolichol chain in relation to translocation of the oligosaccharide-linked precursor [23].

Suggestions for the identity of the putative Lipid II translocase have been given, but so far no experimental evidence has been presented. In a quest to identify the translocator via photocrosslink approach using photoactivatable Lipid II, a specific subset of Escherichia coli proteins was found to be crosslinked, possibly including the translocator, but so far the translocator is not yet identified (unpublished results). However, the discovery in the last decade that lantibiotics specifically dock on Lipid II and thereby kill bacterial cells provides new tools to study Lipid II and its dynamics in the bacterial membrane.

\section{How can lantibiotics in such a versatile manner use Lipid II as receptor to kill bacteria?}

Lantibiotics are a large family of peptides that are produced by certain strains of bacilli to kill other bacteria. The chemical structure of nisin, the best-known and most-studied lantibiotic (Fig. 3), reveals the lanthionine rings, which are a hallmark of the lantibiotics. They are the result of a posttranslational modification in which a dehydrated serine or threonine residue is enzymatically coupled to a cysteine, resulting in ring closure via a thioether bond. The presence of the lantionine rings gives conformational constraints to the polypeptide, resulting in unique three-dimensional structures, different from those that can be adopted by polypeptides of the more regular type.

Nisin is widely used in the food industry as a natural antimicrobial agent. It is active in the $\mathrm{nM}$ concentration range and 


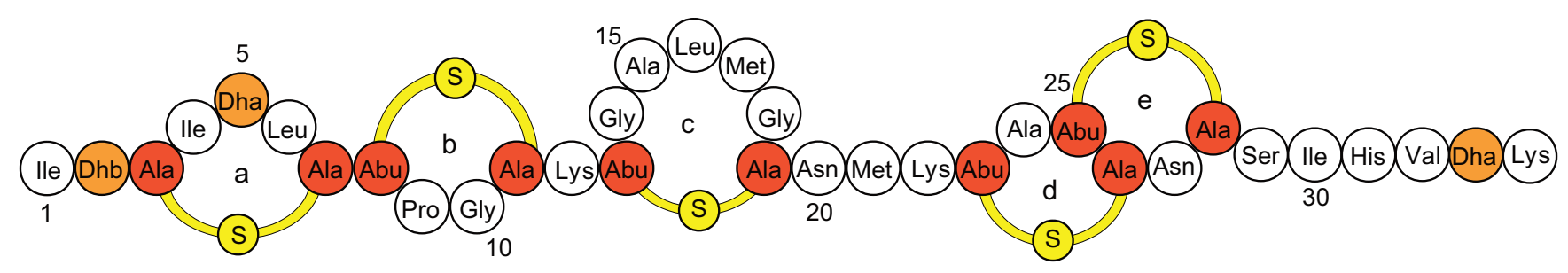

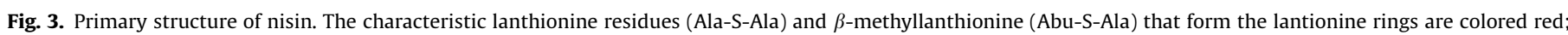
the dehydrated aminoacids Dhb (didehydrobutyrine) and Dha (didehydroalanine) are colored orange.

A

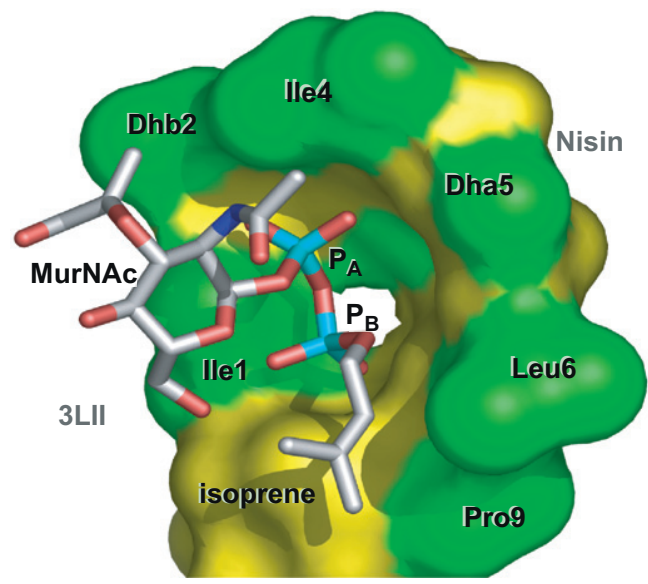

B

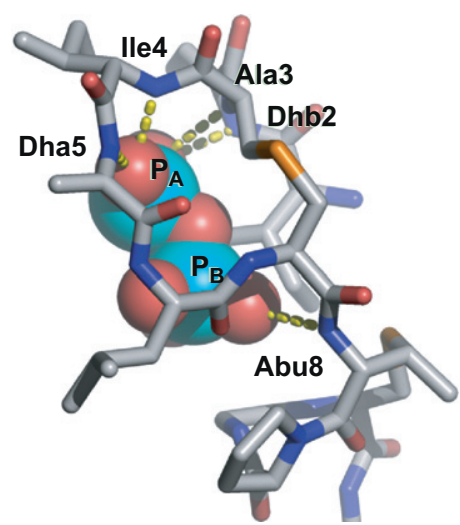

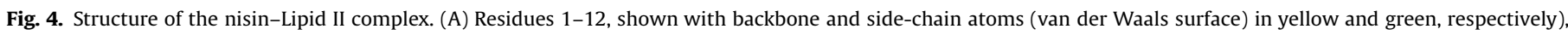

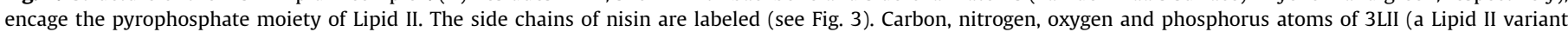

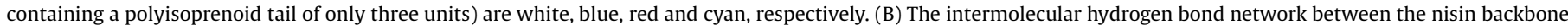

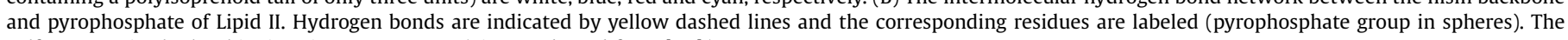
sulfur atoms in the lanthionine rings are orange. (Figure adapted from [26].)

kills bacteria by permeabilizing the plasma membrane, leading to a collapse of vital ion gradients [24]. Efficient permeabilization of the membrane is the result of targeted pore formation. Nisin uses Lipid II as a high-affinity receptor to dock onto the membrane [24]. Lipid II also moderates insertion of nisin into the membrane, which is followed by pore formation [25]. The pores contain both nisin and Lipid II in a defined stoichiometry [26]. The mechanism of action of nisin is now well established and the atomic structure of the nisin-Lipid II complex in solution was determined [27]. The Nterminus of nisin with its characteristic lantinionine ring was found to bind to the pyrophosphate moiety of Lipid II via direct hydrogen bonding with the polypeptide backbone (Fig. 4).

Surprisingly, nisin variants that do not form pores but bind to Lipid II were found to kill bacteria efficiently. Moreover, other lantibiotics that bind, with high affinity, via the N-terminus to Lipid II kill bacteria without permeabilizing the membrane, demonstrating that the lantibiotics have another Lipid-II-mediated mechanism of cell killing [4]. This mechanism was uncovered via fluorescence microscopy techniques, which revealed that lantibiotics that bind Lipid II cluster the molecules into patches when added to phospholipid bilayers, containing small amounts of Lipid II. These studies demonstrated that on the lantibiotic-Lipid II interaction the Lipid II molecule is relocated within the membrane together with the lantibiotic [4]. Since Lipid II was found to be predominantly located into regions of the plasma membrane related to cell elongation and division, this suggested that the lantibiotic might relocate Lipid II out of its functional location and thereby block cell wall synthesis. This hypothesis turned out to be valid. Nisin and also non-pore-forming variants were found to cluster Lipid II into patches in the bacterial cytoplasmic membrane outside the region, where Lipid II is normally present (Fig. 5).
These observations lead to several fundamental questions that are unanswered so far. Lipid II is a fluid membrane lipid that is distributed homogeneously into fluid bilayers but is highly localized in the bacterial membrane. Why is that so? Does this reflect the localization of the cell wall synthesis machinery in which the high affinity of Lipid II for some of its components sequesters Lipid II out of the bulk of the membrane? How do the lantibiotics cluster Lipid II in the membrane? This is probably due to the occurrence of lateral interactions of the lantibiotics in the membrane. But what are these interactions and how are they related to the organization of nisin in the pore complex?

The insights into the mode of action of the lantibiotins, which use Lipid II as receptor, have provided a plausible explanation for the lack of development of major resistance against nisin that is used already so long in the food industry. Nisin binds in a unique manner to the pyrophosphate moiety of Lipid II. It seems unlikely that bacteria can change the pyrophosphate configuration, which appears to be so uniquely designed for Lipid II synthesis and function. In contrast, use of vancomycin, which also binds to Lipid II, but only to the terminal part of the pentapeptide, can result in resistance, because the bacteria can change the composition of the pentapeptide without interfering with cell wall synthesis, thereby blocking the vancomycin-Lipid II interaction and thus losing vancomycin sensitivity. Growing bacteria under pressure of increasing amounts of nisin can develop tolerance to nisin, which is not due to a change in amount or localization of Lipid II but is caused by changes in cell wall thickness and cell wall charge both shielding the plasma membrane from nisin [1].

Another important reason why resistance to nisin will be difficult to develop is that it uses a dual mechanism to kill bacteria. It not only drills a hole in the membrane but it also abducts Lipid II 

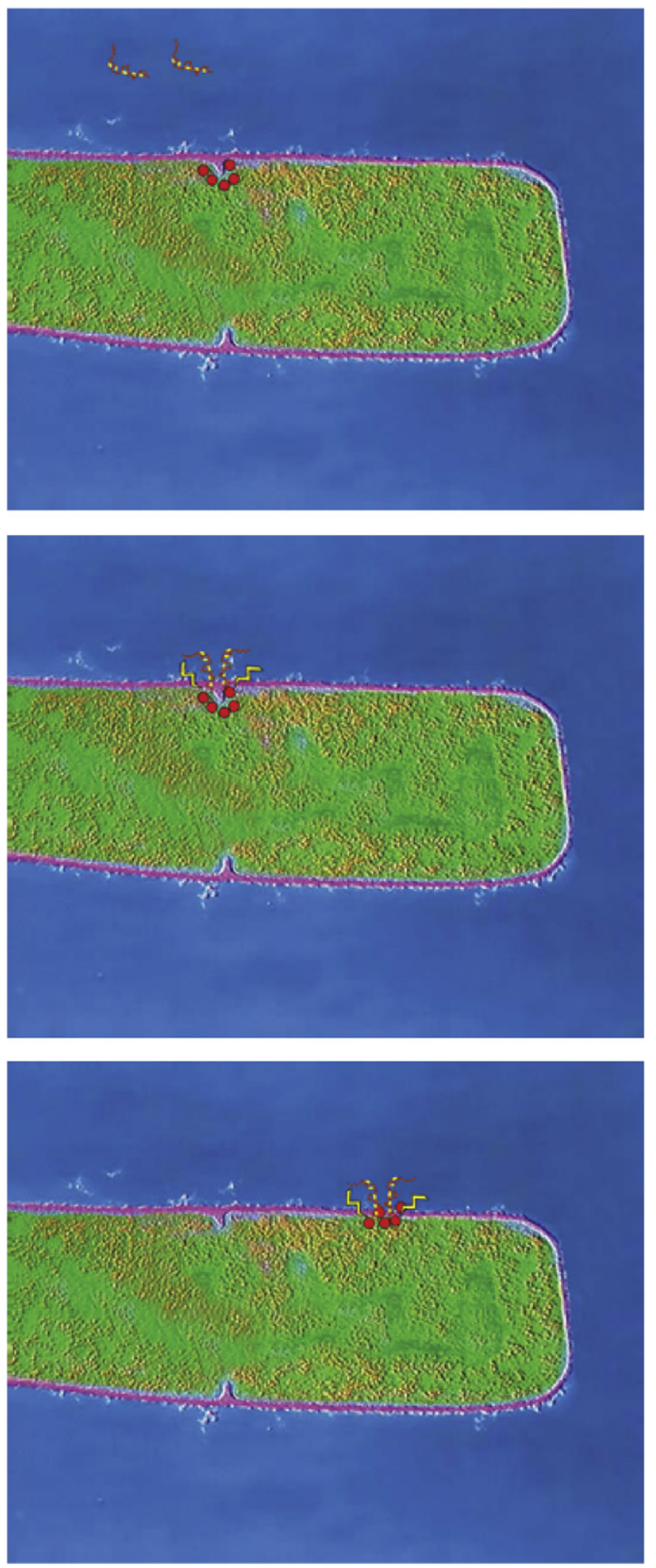

Fig. 5. Model of the segregation of Lipid II by nisin. In a dividing Bacillus subtilis cell (picture adapted from the Science Photo Library with permission), Lipid II is highly localized at the mid-cell region during cell division (top picture, red circles). On binding to nisin (middle picture, red and yellow structure), first pore formation is initiated, resulting in dissipation of the membrane potential (depicted by the lightning bolts). Subsequently, by a yet unknown mechanism, nisin causes Lipid II to be withdrawn from its functional location (the septum) into patches randomly distributed in the membrane (bottom picture). from its functional location, thereby blocking cell wall synthesis. If bacteria were to find a way to oppose one cell killing activity the other one might still prevail. These properties make the lantibiotics fascinating tools for studies in cell wall synthesis but also open up possibilities to develop them into antibiotics of clinical importance.

\section{Acknowledgements}

The authors thank all colleagues, both group members and collaborators who were involved in the studies described in this paper.

\section{References}

[1] N.E. Kramer, et al., Resistance of Gram-positive bacteria to nisin is not determined by lipid II levels, FEMS Microbiol. Lett. 239 (1) (2004) 157-161

[2] E. Breukink, et al., Lipid II is an intrinsic component of the pore induced by nisin in bacterial membranes, J. Biol. Chem. 278 (22) (2003) 19898-19903.

[3] D.N. Ganchev, et al., Size and orientation of the lipid II headgroup as revealed by AFM imaging, Biochemistry 45 (19) (2006) 6195-6202.

[4] H.E. Hasper, et al., An alternative bactericidal mechanism of action for lantibiotic peptides that target lipid II, Science 313 (5793) (2006) 1636-1637.

[5] K.J. Thorne, Identification of prenol intermediates of wall biosynthesis in growing cells of Lactobacillus plantarum, J. Bacteriol. 116 (1) (1973) 235-244.

[6] T.D. Bugg, P.E. Brandish, From peptidoglycan to glycoproteins: common features of lipid-linked oligosaccharide biosynthesis, FEMS Microbiol. Lett. 119 (3) (1994) 255-262.

[7] C.M. Szymanski, et al., Campylobacter-a tale of two protein glycosylation systems, Trends Microbiol. 11 (5) (2003) 233-238.

[8] C. Abeijon, C.B. Hirschberg, Topography of glycosylation reactions in the endoplasmic reticulum, Trends. Biochem. Sci. 17 (1) (1992) 32-36.

[9] P. Burda, M. Aebi, The dolichol pathway of N-linked glycosylation, Biochim. Biophys. Acta 1426 (2) (1999) 239-257.

[10] C.B. Hirschberg, M.D. Snider, Topography of glycosylation in the rough endoplasmic reticulum and Golgi apparatus, Annu. Rev. Biochem. 56 (1987) 63-87.

[11] R. Kornfeld, S. Kornfeld, Assembly of asparagine-linked oligosaccharides, Annu. Rev. Biochem. 54 (1985) 631-664.

[12] S. Hrafnsdottir, J.W. Nichols, A.K. Menon, Transbilayer movement of fluorescent phospholipids in Bacillus megaterium membrane vesicles, Biochemistry 36 (16) (1997) 4969-4978.

[13] R.P. Huijbregts, A.I. de Kroon, B. de Kruijff, Rapid transmembrane movement of C6-NBD-labeled phospholipids across the inner membrane of Escherichia coli, Biochim. Biophys. Acta 1280 (1) (1996) 41-50.

[14] R.P. Huijbregts, A.I. de Kroon, B. de Kruijff, Rapid transmembrane movement of newly synthesized phosphatidylethanolamine across the inner membrane of Escherichia coli, J. Biol. Chem. 273 (30) (1998) 18936-18942.

[15] J.E. Rothman, E.P. Kennedy, Rapid transmembrane movement of newly synthesized phospholipids during membrane assembly, Proc. Natl. Acad. Sci. USA 74 (5) (1977) 1821-1825.

[16] J. Kubelt, et al., Transbilayer movement of fluorescent phospholipid analogues in the cytoplasmic membrane of Escherichia coli, Biochemistry 41 (17) (2002) 5605-5612.

[17] R.D. Kornberg, H.M. McConnell, Inside-outside transitions of phospholipids in vesicle membranes, Biochemistry 10 (7) (1971) 1111-1120.

[18] M.A. Kol, et al., Membrane-spanning peptides induce phospholipid flop: a model for phospholipid translocation across the inner membrane of E. coli, Biochemistry 40 (35) (2001) 10500-10506.

[19] M.A. Kol, et al., Phospholipid flop induced by transmembrane peptides in model membranes is modulated by lipid composition, Biochemistry 42 (1) (2003) 231-237.

[20] A. Zachowski, Phospholipids in animal eukaryotic membranes: transverse asymmetry and movement, Biochem. J. 294 (Pt 1) (1993) 1-14.

[21] V. van Dam, et al., Transmembrane transport of peptidoglycan precursors across model and bacterial membranes, Mol. Microbiol. 64 (4) (2007) 1105-1114.

[22] J. Helenius, M. Aebi, Transmembrane movement of dolichol linked carbohydrates during $\mathrm{N}$-glycoprotein biosynthesis in the endoplasmic reticulum, Semin. Cell Dev. Biol. 13 (3) (2002) 171-178.

[23] M.A. McCloskey, F.A. Troy, Paramagnetic isoprenoid carrier lipids. 2. Dispersion and dynamics in lipid membranes, Biochemistry 19 (10) (1980) 2061-2066.

[24] E. Breukink, et al., Use of the cell wall precursor lipid II by a pore-forming peptide antibiotic, Science 286 (5448) (1999) 2361-2364.

[25] H.E. van Heusden, B. de Kruijff, E. Breukink, Lipid II induces a transmembrane orientation of the pore-forming peptide lantibiotic nisin, Biochemistry 41 (40) (2002) 12171-12178.

[26] H.E. Hasper, B. de Kruijff, E. Breukink, Assembly and stability of nisin-lipid II pores, Biochemistry 43 (36) (2004) 11567-11575.

[27] S.T. Hsu, et al., The nisin-lipid II complex reveals a pyrophosphate cage that provides a blueprint for novel antibiotics, Nat. Struct. Mol. Biol. 11 (10) (2004) 963-967. 